Systematic Review

\title{
A Systematic Review on the Prevalence, Etiology, and Pathophysiology of Intrinsic Pain in Dermal Scar Tissue
}

Eveline Bijlard, MD', Lisa Uiterwaal, MD', Casimir A. E. Kouwenberg, MD', Marc A.M. Mureau, MD, PhD', Steven E. R. Hovius, MD, PhD', and Frank J. P. M. Huygen, MD, PhD²

From: ${ }^{1}$ Department of Plastic and Reconstructive and Hand Surgery. Erasmus MC, Erasmus University Medical Centre Rotterdam, The Netherlands; 2Department of Anesthesiology, Pain Treatment Centre. Erasmus MC, Erasmus University Medical Centre Rotterdam, The Netherlands

Address Correspondence: Eveline Bijlard, MD Plastic Surgery, Erasmus MC, Faculty EE1591, PO Box 2040, 3000 CA Rotterdam, The Netherlands

E-mail: e.bijlard@erasmusmc.nl

Disclaimer: There was no external funding in the preparation of this manuscript. Conflict of interest: Each author

certifies that he or she, or a member of his or her immediate

family, has no commercial association (i.e., consultancies, stock ownership, equity interest, patent/licensing arrangements, etc.) that might pose a conflict of interest in connection with the submitted manuscript.

Manuscript received: 04-19-2016 Revised manuscript received: o8-01-2016 Accepted for publication: 09-16-2016

Free full manuscript: www.painphysicianjournal.com
Background: Scars can cause pain, even without symptoms of underlying nerve damage. A lack of knowledge on intrinsic scar pain hampers effective treatment of these complaints.

Objective: Aggregate current knowledge on the prevalence, etiology, and pathophysiology of intrinsic pain in dermal scars.

Study Design: Systematic review.

Setting: University Medical Center.

Methods: We searched the Embase, Medline, Cochrane central, CINAHL, Web-of-Science, and Pubmed databases with search terms: scar, skin, pain, and etiology/pathology, adding all synonyms of these terms. Relevant papers were selected and analyzed by 3 reviewers.

Results: Intrinsic pain in scars has a low prevalence. However, pathologic scars and burns regularly cause pain of high intensity. The etiology is multifactorial, the extent of trauma was an important predicting factor. Nerve fiber density did not explain the intrinsic pain when panneuronal markers were used, while a correlation with an increased number of C-fiber subtypes seems plausible. Nerve growth factor (that stimulate these C-fibers) plays an important role in wound healing. Thereby, it also sensitizes neurons and promotes inflammation, releasing even more neurotrophic factors. Central sensitization causes a long-lasting effect even after wounds are healed. Furthermore, the opioid-system, that influences inflammation and healing and possible systemic sensory alterations after injury, is discussed.

Limitations: Liberal selection criteria challenged the systematic selection of papers.

Conclusions: Burn and pathologic scars often lead to high intensity pain symptoms. This pain has many characteristics of neuropathic pain that could be caused by an imbalance of C-fibers subtypes. The scar tissue itself may alter the nerve fiber distribution; the imbalance results in ongoing neuro-inflammation and pain symptoms.

Key words: Systematic review, scar, pain, epidermal innervation, prevalence, neuro inflammatory response, peptidergic fibers

Pain Physician 2017; 20:1-13

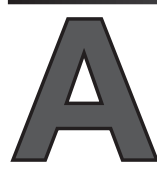
fter injury our skin heals by forming scar tissue. Dermal scars can decrease quality of life by esthetic, psychological, and physical complaints. The latter consists of movement restriction, itch, and pain, of which pain gives rise to the most severe burden $(1,2)$. Burn patients and patients with pathologic scars often visit dermatologists or plastic surgeons, who often experience difficulties treating the pain complaints. This leaves these patients without proper treatment. 
A better understanding of the mechanisms involved in painful scars and their treatment options could improve pain relief for these patients.

Wounds heal by a complex process involving several phases. During the inflammatory phase, which starts directly after injury, a blood clot is formed and pro-inflammatory cytokines produced by cells like macrophages and mast cells attract neutrophils to the wound. Around the fourth day after trauma, debris and bacteria are removed by the macrophages and neutrophils; furthermore, angiogenesis starts and fibroblasts get activated. Hereafter, the proliferative phase lasts for around 2 to 6 weeks. Several growth factors stimulate the fibroblasts and keratinocytes. Angiogenesis makes growth of granulation tissue possible and epithelialization takes place. After the wound is completely closed, the scar matures for approximately one year. New tissue with collagen fibers arranged at random transforms to a well-organized network under mechanical stress adding strength to the scar. This occurs by a continuous process of degradation and generation of collagen (3).

A normotrophic scar appears as a thin white line in plane with the surrounding skin. Scars can present with different, abnormal phenotypes, like atrophic scars (stretched out and thinner than surrounding skin), contracted scars (shorter than the original wound), hypertrophic scars (raised, red, and itching or painful), or keloids (growing into surrounding skin and forming discolored and itching or painful tumors). Abnormal scar types are most likely the result of dysregulation in the wound healing process, but the exact mechanisms are not clarified yet.

Pain is a common symptom during wound healing and it generally occurs in the initial phase as a result of tissue damage. Pain complaints generally fade during the phases of wound healing and cease in the maturation phase of healing when a scar has formed. If a matured scar is still painful, one possible cause of this pain is a neuroma, which originates from a regenerating nerve trapped in fibrotic dermal scar tissue. Neuromas have a typical clinical presentation (positive Tinel sign, numbness in the innervation area of the injured nerve) and treatment can be directed to eliminate the neuroma (4). Unfortunately, some patients have painful scars without the typical symptoms accompanying a neuroma. The prevalence of pain symptoms in different scar types like hypertrophic scars and keloids and the mechanisms behind them are largely unknown (2). In order to generate an effective treatment against painful scars we need to know the extent of the problem and the underlying mechanisms causing pain. Therefore, we aimed to perform a comprehensive systematic review on the prevalence and intensity of pain, and the knowledge of pathophysiology and etiology of pain in dermal scars.

\section{Methods}

\section{Literature Search Methods}

We searched the Embase, Medline, Cochrane central, CINAHL, Web-of-Science, and Pubmed databases. In each database, we used the following search terms: scar, skin, pain, and etiology/pathology, adding all synonyms of these terms. The full search terms used can be found in the appendix. The search was performed from inception of the databases until December 2, 2014.

We included all English articles that mentioned the prevalence, intensity, or a cause of pain in dermal scar tissue. To exclude pain caused by wounds and neuromas, we used the following definition of a painful scar: persistent pain in a healed scar over 3 months' duration, with allodynia and hyperalgesia adjacent to the scar, and with no sensory loss other than over the scar itself (5). In order to include all relevant information we also selected reviews. We excluded papers solely based on the treatment of (painful) scars, scars in other than dermal tissue, neuromas, and papers otherwise irrelevant to the research question.

First, to select relevant papers 2 reviewers (EB, LU) independently assessed titles and abstracts. In cases of disagreement, a third reviewer (CK) also assessed title and abstract and made the final decision on eligibility. To make a final decision about inclusion, the full-text of the eligible articles were assessed by 2 reviewers (EB, LU), if there was no consensus the article was discussed by the reviewers until consensus was reached. The references of all selected articles were crosschecked and relevant papers were included (Fig. 1).

\section{Literature Analysis Methods}

For all included papers, 2 reviewers (EB, LU) independently extracted relevant information to answer our research question. We searched data on: 1) prevalence and intensity of pain in all types of dermal scars; 2) etiology, what biological, psychological, or environmental factors aggravate pain in dermal scars; and 3) pathophysiology, what is the underlying biological mechanism that causes pain in some scars and not in others. The collected information of both reviewers was verified by the other reviewer. 
The papers that described pain intensity used different instruments, often the visual analog scale (VAS, range $0-10$ ) or numeric rating scale (NRS, written or verbal, range $0-10$ ) were used, if other scales were used (i.e., scores ranging $1-5$ ) scores were converted to a 0 - 10 scale to improve comparability between papers.

\section{Results}

Our search resulted in 2,049 records, and after completion of the entire selection process, 52 papers remained with relevant information about painful scars (Fig. 1).

\section{Epidemiology}

We found 18 papers with reference to the prevalence of scar pain and pain intensity (Table 1) (6-23). Most studies used questionnaires not suitable for differentiating between neuromas and painful scars. Many studies investigated a specific subgroup of dermal scars with a high prevalence of pain symptoms, mainly burn scars. These studies did not report the prevalence of pain in other scar types.

Of the 14 papers that reported on pain prevalence, 8 used a questionnaire, of which only 4 included validated questionnaires. Others used merely a VAS scale, single questions, or chart notes. Of the patients with a burn scar $25 \%-68 \%$ suffered from pain. However, after reviewing the results from these studies, nerve damage underneath the scar could not be ruled out. Scar pain after surgery with a low chance of nerve damage occurred in about $10 \%$ of patients, with $2 \%$ experiencing substantial pain (>3/10). Surgery with a higher risk of nerve damage, like amputation and thoracotomy, more often caused painful scars $(30 \%-50 \%)(12)$. Many of these patients ( $80 \%$ as described by Hoimyr et al [10]) showed sensory disturbances distal to the scar, suggesting nerve damage. These results indicate a prevalence of painful scars without nerve damage in less than $2 \%$ of patients.

Fourteen papers reported on pain intensity; in 5 papers pain intensity was recorded with a self-completed anchored VAS ranging from $0-10 \mathrm{~cm}$. Four other papers used a NRS from 0 - 10 or from $1-10$. The intensity of pain reported by burn patients varied from 1.3 to 5.6 on a 10 -point scale. Patients with hypertrophic scars reported a lower intensity of pain (2.2) compared to patients with keloids $(5.4-6.3)$ (Table 1$)$. Patients experience pain from their scars, while sensibility and pain thresholds in the scars are raised when they are objectively tested $(16,22)$. Some studies found that pain reduced with time (15), while other studies could not confirm this (17).

None of the studies did a validated quality of life assessment to assess the burden of a scar, but some did inquire about impairment of daily life functioning. For surgical scars, Hoimyr et al (10) found $6.6 \%$ of patients

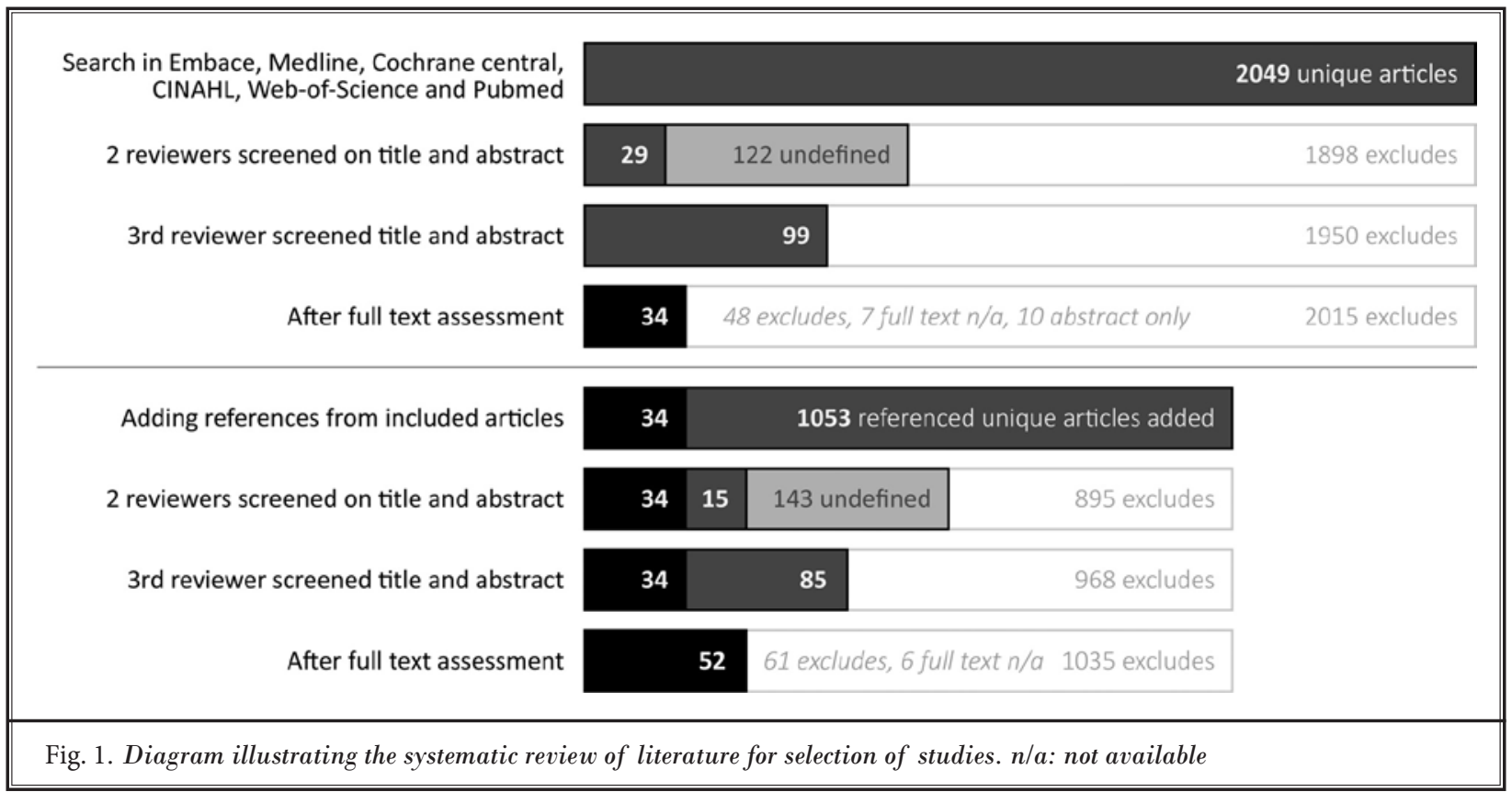




\begin{tabular}{|c|c|c|c|c|c|c|c|c|c|c|c|c|c|c|c|}
\hline 昜 & 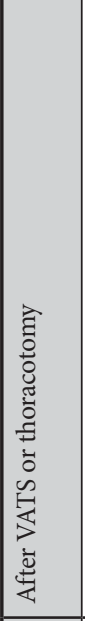 & 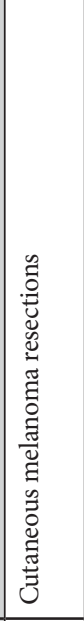 & 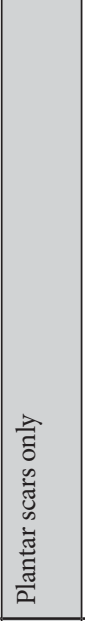 & 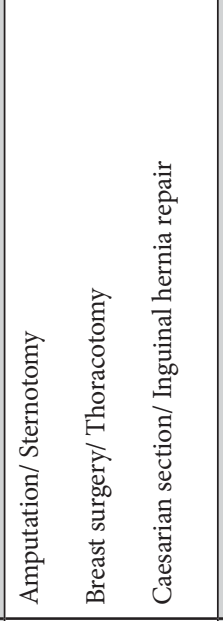 & 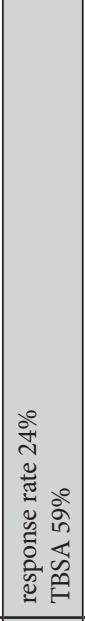 & 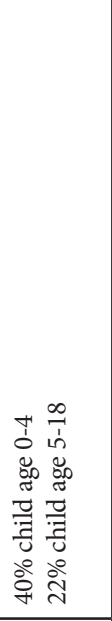 & 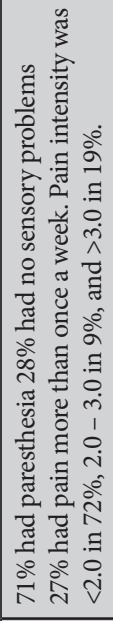 & 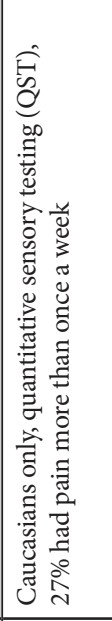 & 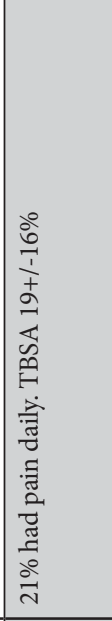 & 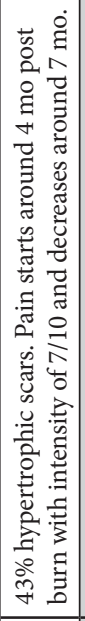 & 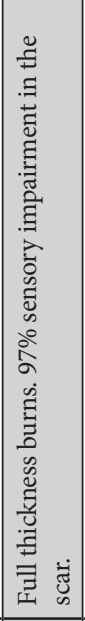 & 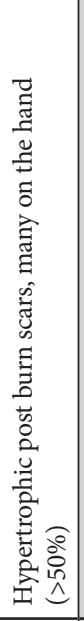 & 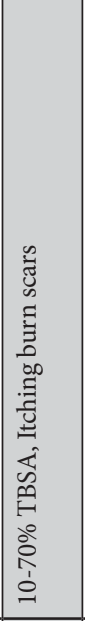 & 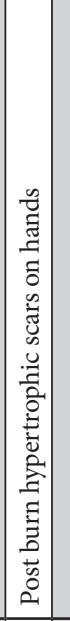 & \\
\hline 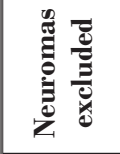 & z & z & $\ddot{z}$ & $\stackrel{\circ}{z}$ & z & 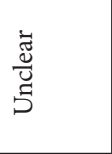 & \& & z & $\stackrel{0}{z}$ & \begin{tabular}{|l}
$\overrightarrow{\tilde{J}}$ \\
$\overrightarrow{\breve{g}}$ \\
5
\end{tabular} & 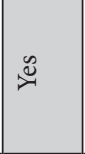 & $\cong$ & 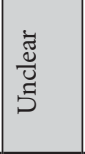 & $\approx$ & $\stackrel{8}{z}$ \\
\hline 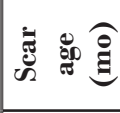 & $\begin{array}{l}\not \\
\infty \\
i \\
i\end{array}$ & 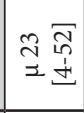 & \begin{tabular}{ll|}
$\vec{m}$ & $\bar{b}$ \\
2 & 0 \\
& 0
\end{tabular} & ' & $\begin{array}{|ll|}0 & \overline{2} \\
0 & 0 \\
z & 0 \\
\end{array}$ & $m \in \simeq$ & 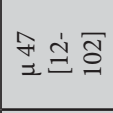 & $\stackrel{8}{:} \stackrel{\infty}{\Xi} \bar{\Xi}$ & $\underset{\substack{\widehat{N} \\
=}}{\stackrel{\mathbb{I}}{\Xi}}$ & $\ddot{\wedge}$ & 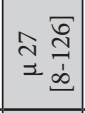 & 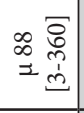 & 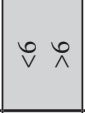 & . & ' \\
\hline 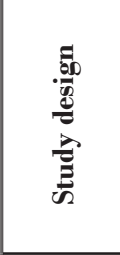 & 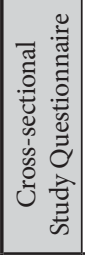 & 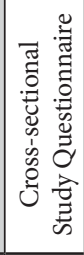 & 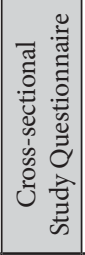 & 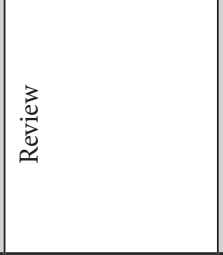 & 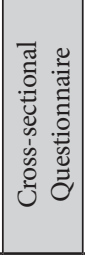 & 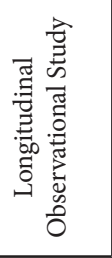 & 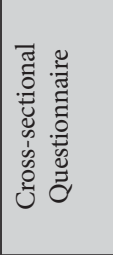 & 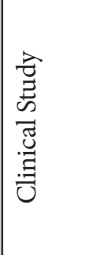 & 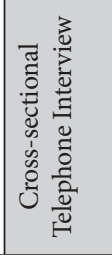 & 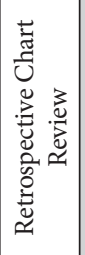 & 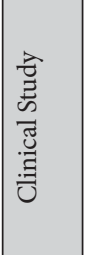 & 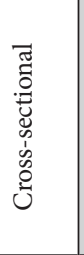 & 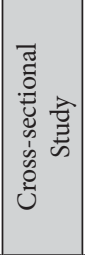 & 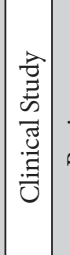 & 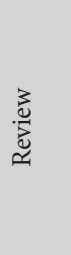 \\
\hline 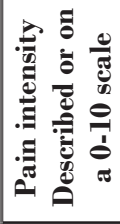 & 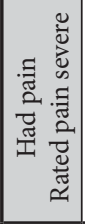 & 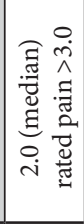 & 吾 & 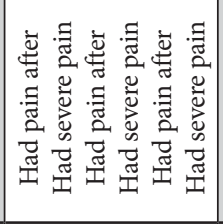 & & 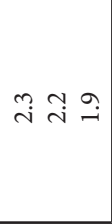 & 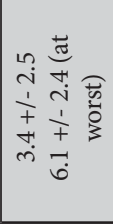 & 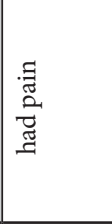 & 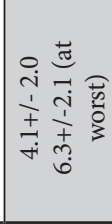 & & & $\begin{array}{l}\vec{i} \\
i \\
i \\
+ \\
i \\
i\end{array}$ & 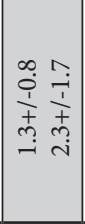 & 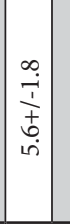 & \\
\hline 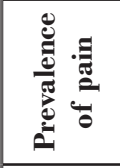 & 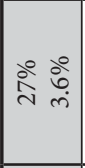 & 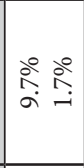 & 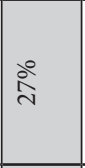 & 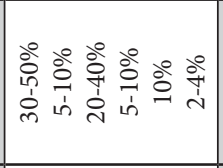 & iิ & & ठें & 今े & 今ें & 高 & ओे & & & مें & ळे \\
\hline 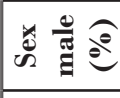 & 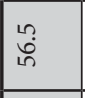 & $\vec{A}$ & $\stackrel{4}{\dot{n}}$ & , & in & $\overrightarrow{6}$ & 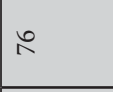 & $\infty$ & $\stackrel{0}{1}$ & 6 & 22 & 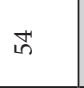 & $\overrightarrow{0}$ & $\infty$ & ' \\
\hline 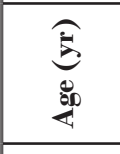 & in $\begin{array}{c}\sqrt{6} \\
\text { in } \\
\stackrel{+}{=}\end{array}$ & $\begin{array}{l}0 \\
+1 \\
+1 \\
0 \\
0 \\
0\end{array}$ & 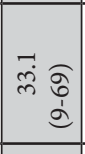 & ' & F $\widehat{\widehat{\sigma}}$ & $\begin{array}{l}0 \\
+1 \\
0 \\
n \\
\text { in }\end{array}$ & 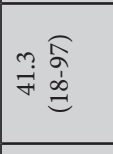 & 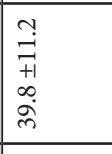 & 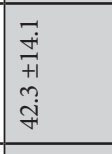 & 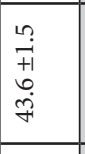 & 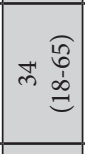 & 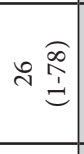 & $\stackrel{\infty}{\wedge}$ & $\begin{array}{l}2 \\
\tilde{z} \\
\infty \\
\infty \\
\dot{q}+\end{array}$ & ' \\
\hline z & 8 & शे & $m$ & ' & in & ભે & $\underset{\sim}{0}$ & $\vec{I}$ & 节 & in & 8 & m & 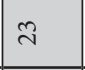 & $\pi$ & \\
\hline 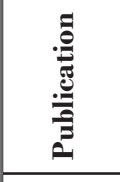 & 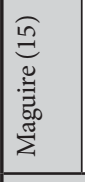 & 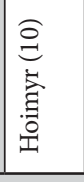 & 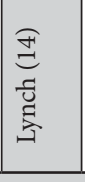 & 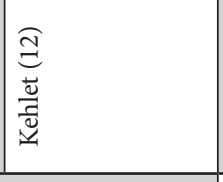 & 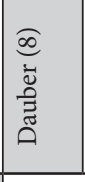 & 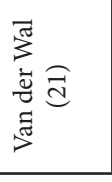 & 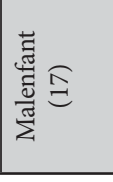 & 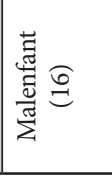 & 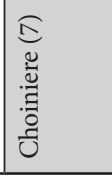 & 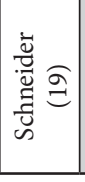 & 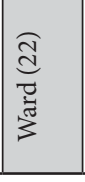 & $\begin{array}{l}\text { बa } \\
\ddot{d} \\
\text { d }\end{array}$ & 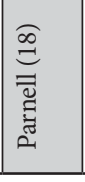 & 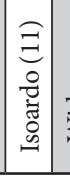 & 竞 \\
\hline & \multicolumn{4}{|c|}{ 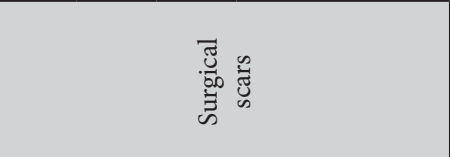 } & \multicolumn{11}{|c|}{ 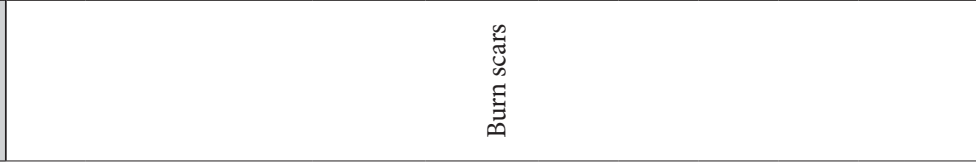 } \\
\hline
\end{tabular}




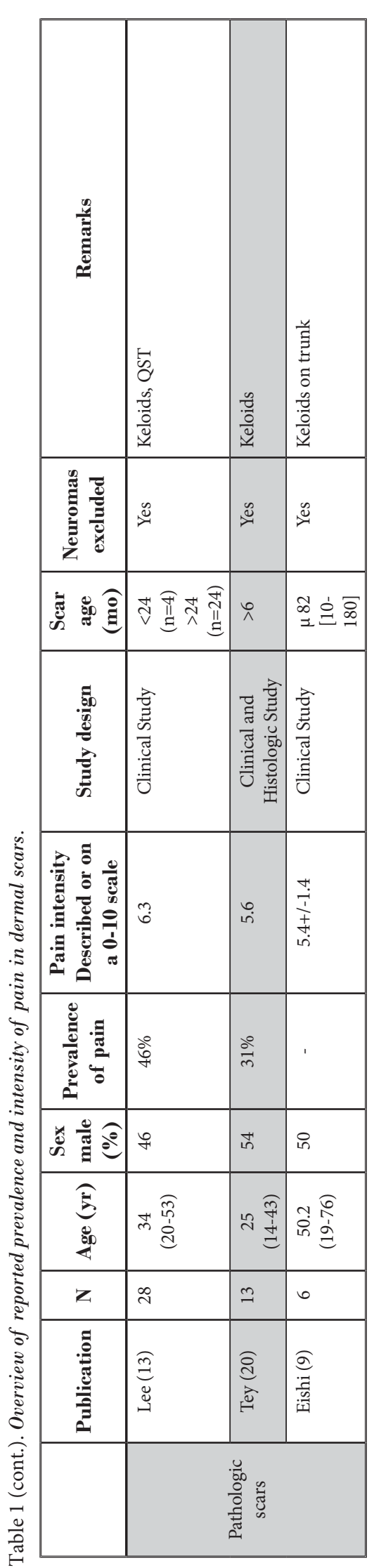

that had a surgical scar had pain that impacted their daily life, while Maguire et al (15) found $18 \%$ of the patients after thoracotomy ( $40 \%$ of patients with pain) had pain that limited daily activities. In studies looking at burn scars several aspects are described like difficulties sleeping or problems performing work or social activities; prevalence ranges from $10 \%$ to $20 \%$ with exception of Choiniere et al (7) and Dauber et al (8) who show a prevalence of $45 \%$ and $55 \%-75 \%$, respectively, on these topics $(7,8,17-19)$.

In summary, pain in dermal scars without nerve damage appears to have low prevalence among patients with scars (12), while specific subgroups of scars, like pathologic scars and burns, regularly cause pain. Patients with pathologic scars are also affected by pain of higher intensity.

\section{Etiology}

We found different factors that raise the risk of painful scars. Like many conditions, painful scars are considered multifactorial and genetic susceptibility has its role (although no specific genes are identified). In post-surgical scars, the surgical procedure and technique are evidently of influence $(12,14,15)$. Further, younger patients are more prone to develop painful scars after surgery $(10,12,15)$. We found contradicting results on whether the length of time after surgery influences pain $(10,15)$. However, the length of time after burn injury did not reduce pain $(7,16,17,22)$. In burns, size and the depth of the burn predicted painful burn scars in most studies $(7,16,17,21)$. In contrast to post-surgical scars, there was no relation between post-burn pain and age $(11,17,21,22)$. Kehlet et al (12) state that women are more often affected with painful scars, but others found no difference with gender $(7,10,11,15-17,24)$.

Another possible factor related to pain after injury and scar formation is traumatic memories associated with the scars. Both anxiety and stress correlate well with the perception of pain sensations $(10,25,26)$. Pre-operative anxiety is correlated with post-operative pain experience. However, although pre-operative catastrophizing scores (tendency to exaggerated pessimism on outcome) correlated with acute post-operative pain, they did not correlate with chronic post-operative pain or long-lasting painful scars (12). Conditions associated with neuropathic post-burn pain are post-traumatic stress disorder, substance abuse, and depression (19). 


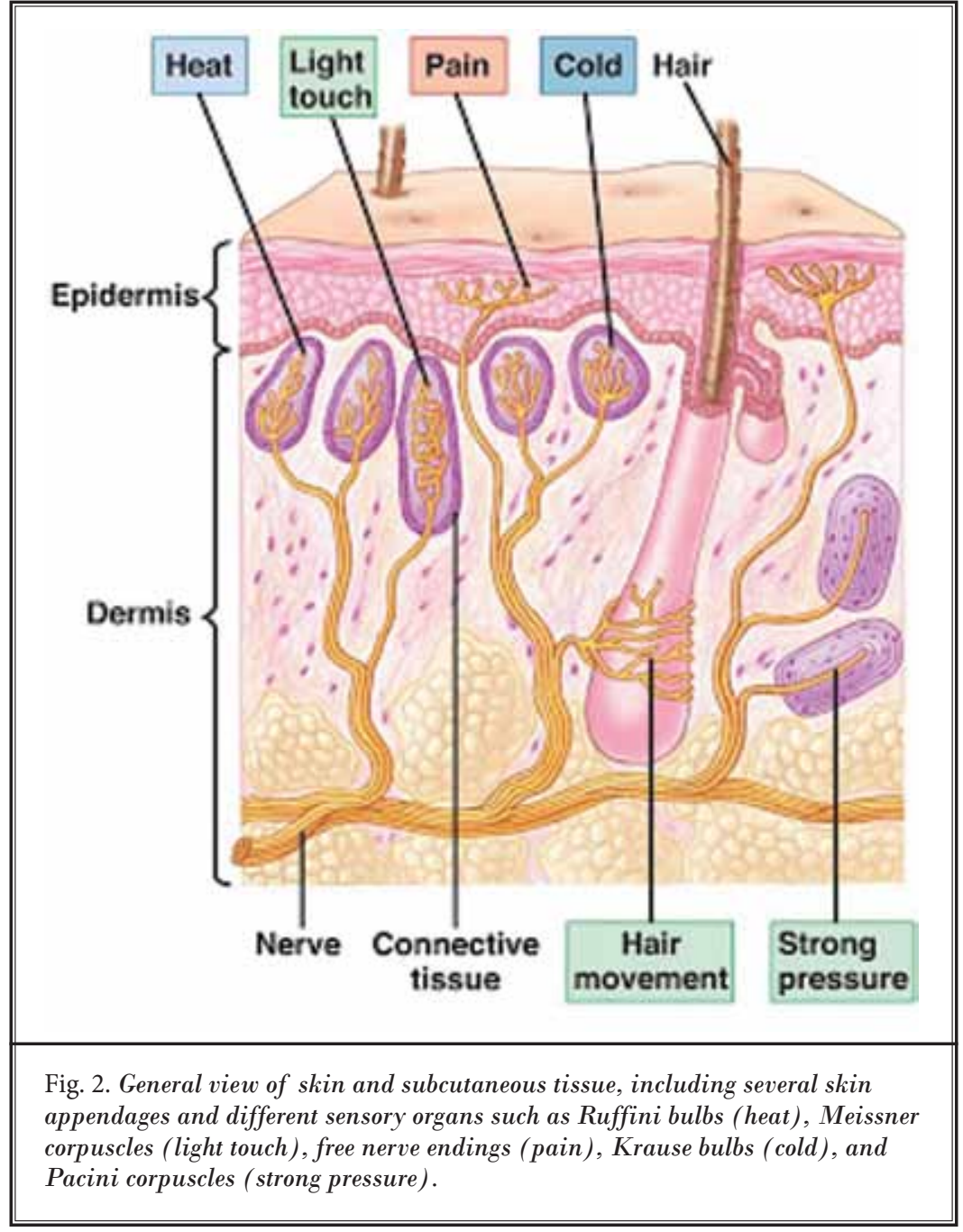

\section{Pathology}

Most studies retrieved from the search studied the underlying mechanism of painful scars.

Numerous specialized structures are present in the skin to detect various stimuli. Merkel cells in the epidermis and Meissner corpuscles in the dermal papillae both are able to detect light touch. Pacini corpuscles, which are specialized to detect pressure, are found deep within the dermis or even in the subcutaneous tissue. Pain is transmitted through naked nerve endings located in the basal layer of the epidermis. Krause bulbs detect cold, whereas Ruffini corpuscles detect heat. Heat, cold, and proprioception also are located in the superficial dermis (Fig. 2).

Free nerve fibers are responsible for pain transmission. They consist of both fast transmitting, myelinized A $\delta$-fibers and slower, unmyelinized C-fibers. Nerves fibers are present all over the skin. The density of these fibers differs per body area, varies between individuals and between childhood and adulthood. Another but a very relevant factor is the technique that is used to visualize the fibers. This makes it hard to directly compare results between tests and studies, and only ratios and conclusions can be used to compare outcome. In neuropathic disease fiber density can be affected $(27,28)$.

\section{Nerve Fiber Density}

Abnormal nerve fiber density has often been suggested as the cause for painful scars, but evidence is conflicting and weak (Table 2) $(20,29-40)$. Many differences found in fiber density can be attributed to differences in methods of fiber staining, different skin layers, scar types, and scar age $(33,34,36,37,41)$. Nonetheless, several factors may increase nerve sprouting, such as neonatal age and low opioid receptor availability $(38,42)$. High nerve fiber density can cause pain by inappropriate cross-stimulation as receptors are in much closer proximity, resulting in central sensitization (34). However, most painful peripheral neuropathies show decreased fiber density, as in diabetic neuropathy or HIV (43).

Skin nociception is transmitted through myelinated, fast conducting $A \delta$-fibers that transmit sharp acute pain and by unmyelinated slow conducting C-fibers that represent about $70 \%$ of all epidermal fibers and conduct diffuse, burning, aching, and dull type pain. In general, normotrophic matured scars seem to have less innervation than normal skin $(30,33,37,39-41,44)$. Pathologic and painful scars seem to have richer innervation than normal skin, as shown by an increased nerve fiber density, specifically in the peptidergic (substance P [Sub-P] or calcitonin gene-related peptide (CGRP) immunoreactive [IR]) fibers $(33,34,36,39-41,45)$. The C-fibers are generally divided in peptidergic and non-peptidergic fibers, the former 


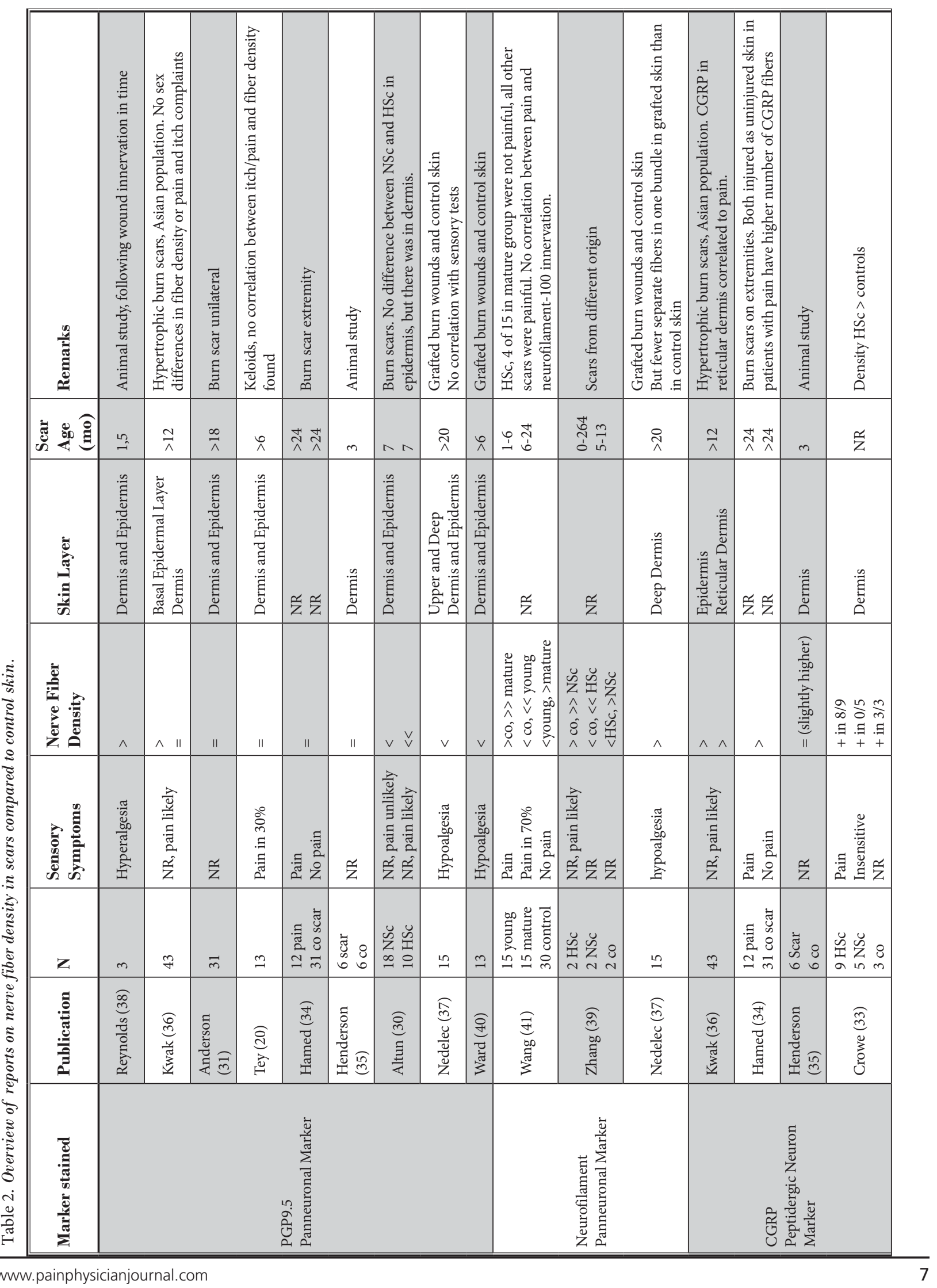




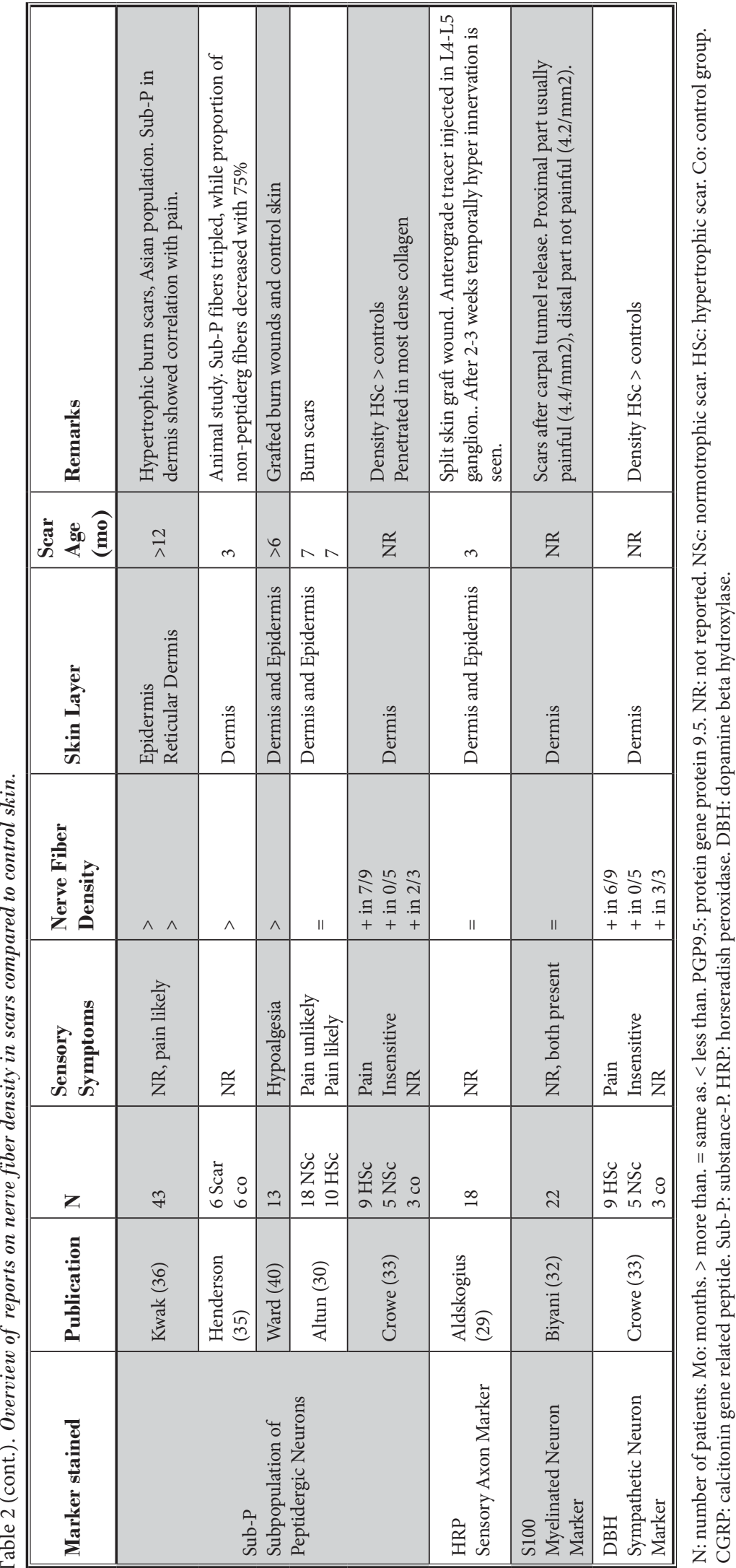

are CGRP and Sub-P IR and respond to nerve growth factor (NGF), while the latter is P2X3 and IB4 IR and responds stronger to glial derived neurotrophic factor (GDNF), the functional difference between these types of C-fibers is not yet clarified. However, other groups found lower or no change in nerve fiber density in painful scars $(30,32,46)$.

The natural course of wound healing results in increased innervation in the first weeks of scar formation, gradually returning to normal or lower levels $(29,35,41,45)$. A disruption in the physiologic decrease of nerve fibers might cause painful scars. For example, Henderson et al (35) showed that Sub$P$ remains elevated for more than 3 months after trauma. At that moment the scar contains $89 \%$ peptidergic fibers (compared to $57 \%$ in uninjured skin), which provide pain transmission. This illustrates the disturbed balance between peptidergic and non-peptidergic fibers, which is supported by several studies $(30,35,40)$.

Pain symptoms did not correlate with nerve fiber density consistently when pan-neuronal markers were used (PGP9.5, S100), while a correlation with an increased number of peptidergic fibers (CGRP-IR/Sub-P-IR), either absolute or relative, is thought to be likely $(34,47)$. Clinical sensibility studies in keloid scars showed deficits matching with small fiber neuropathy (which is characterized by a lower epidermal nerve fiber density) (13). However, in burn scars a fiber specific sensibility impairment was not found (16).

Finally, independent of nerve fiber density, damage or mechanical compression of $\mathrm{A} \delta$-fibers and $\mathrm{C}$-fibers by dense scar tissue can also be the cause of pain $(11,26)$.

\section{Neurotrophic Factors}

NGF is an important neurotrophic factor that is required to keep neurons viable. NGF is produced by Schwann cells, but also by keratinocytes at the 
basal-epidermal junction of the skin. NGF is present in high concentrations during wound healing as it stimulates keratinocytes to migrate until contact to other keratinocytes is re-established. NGF also stimulates melanocytes to form dendrites to transport pigment that colors the skin (5). Both wounds that took longer to re-epithelize and persons with pigmented skin had higher risk on making pathologic scars and might have had higher levels of or prolonged NGF exposure (2).

Besides its functions in the skin, NGF triggers neuronal sensitization in several ways. First, NGF directly affects primary sensory neurons, resulting in hyperexcitability of the neurons (48). Second, NGF stimulates sympathetic neurons to produce more neurotransmitters to the branches of these fibers that are in close proximity to the cutaneous nociceptors $(5,48,49)$. Third, it also activates mast cells, lymphocytes, and leucocytes, which release inflammatory factors as Sub-P, CGRP, and platelet activating factor. CGRP has direct stimulating effects on nociceptors and potentiates the effects of other factors. Platelet activating factor releases serotonin from the platelets. Serotonin injected in skin causes pain at the injection site, and in hypertrophic and red scars histamine, as well as serotonin levels, are increased (50). Increased vascularization and red appearance of scars correlated with more pain symptoms reported by patients (51). The inflammatory response stimulates the primary sensory nerves in many ways. On the other hand, while Choi et al (6) found more mast cells in scar tissue than in control skin, they found no correlation between mast cells and pain or between itch and pain.

The amount of NGF produced by keratinocytes is sufficient to affect neuronal growth and pain behavior. Experimental studies with laboratory animals showed that damaged nerve fibers near keratinocytes caused local NGF levels to rise. This resulted in directed and abundant sprouting and hyperexcitability of the damaged axons, resulting is clear neuropathic pain behavior $(52,53)$. Persistent elevated NGF levels and persistent inflammation can cause permanent hyperinnervation and hyperalgesia $(5,48)$. This could explain why prolonged wound healing and continued release of inflammatory substances (interleukine-1, tumor necrosis factor-alfa) more often results in symptomatic pathologic scars $(12,48,54)$. Altering this response has been tried in several ways, starting as simple as preventing stimulation of free nerve endings to reduce neuropeptide and inflammatory substance release. An occlusive dressing, for example, can reduce pain and scar tissue formation (23).
In summary, NGF plays an important role in wound healing and therefore scar formation, but it also sensitizes neurons and promotes inflammation, a process that releases other neurotrophic factors. When the sensitization is distinct this is a long lasting effect, and complaints can be present after the skin restored its continuity.

\section{Pain and Itch in Scars}

Pain and itch often co-exist in pathologic and burn scars, possibly because their mechanisms are closely related. Itch is partly transmitted by C-fibers, which also transmit pain. It is hypothesized that weak stimuli of C-fibers produces itch and stronger stimulus produces pain sensation $(26,47)$. Mast cell degeneration can lead to itch as well as pain by releasing histamine, leukotrienes, Sub-P, prostanoids, and growth factors that activate peptidergic C-fibers $(9,45,47)$. All these factors result in neuroinflammation that causes itch or pain. Histamine levels are high in young burn scars and return to normal levels when the scar matures $(45,50)$.

Another mechanism that influences itch and pain is the opioid system. Opioid mediated regulations are widely expressed in the central nervous system, but opioid receptors (MOR, KOR, and DOR) are also expressed in the skin where they influence skin homeostasis and pain and itch $(42,55)$. Nerve fiber density (PGP9.5) was increased and fiber morphology was changed in MOR and KOR knockout mice. It seems likely that the opioid system caused these effects, because there were no apparent signs of increased inflammation (like increased mast cell count and CD4+ count) (42). In humans, opioid antagonists can reduce itch that does not respond to antihistamines, but may give rise to pain. Opioids reduce pain and can induce itch, which hardly responds to either opioid antagonists or antihistamines, indicating that opioid induced pruritus is not solely transmitted by histamine $(47,48,55,56)$. In pathologic scars MOR, KOR, and DOR were all increased, as was anti-nociceptive beta-endorfin (MOR ligand). This activation influences peripheral nociception and pruritus $(47,55,56)$. Longterm opioid use increases pro-inflammatory cytokines in an existing wound, while opioids administered before surgery diminish pro-inflammatory response (55). Use of opioids impaired scar strength after secondary healing by inhibition of neo-angiogenesis. However, it increased strength of incisional wounds by enhanced scar remodeling, with up-regulation of transforming growth factor-beta and metametalloproteinase-2 (57).

In summary, opioids influence inflammatory re- 
sponse and wound healing; the direction is dependent on wound type and opioid timing $(55,57)$. The coexistence of itch and pain that are both affected by the opioid system, does warrant further research in this direction.

\section{Systemic Effects}

Although scars are well-bounded local lesions, several reports suggested that not only local processes play a role in pain perception. Studies investigating sensory functions and nerve fiber density using both the contralateral side and other participants as controls, found sensory functions were not only disturbed at the scar area but also at the uninjured contralateral skin $(16,31,34,46)$.

Aberrant sensory functions and increased vulnerability to pain are long-term complications after neonatal surgery and these effects are not restricted to the scar area $(58,59)$. It seems that the developing peripheral nerve system in neonates is highly influenced by painful stimuli. This could affect synaptic connectivity in the central nervous system that may have long-lasting effects $(38,58)$.

In adults, nociceptor activity in burn wounds can activate dorsal horn microglia. This activation is key to neuropathic pain development and will also affect pain perception in skin adjacent to the scar (60). Patients with chronic pain after burn injury have a high amount of CGRP-IR fibers in both the scar and uninjured skin, while in patients without pain, the scar and uninjured skin both have few CGRP-IR fibers (34). An animal study on burn wounds showed nerve fiber density (PGP9.5) was diminished 2 weeks after injury. This effect was seen at both the injured and non-injured site, albeit stronger at the injured site. This decrease in nerve fiber density was present until 12 weeks and maybe lasted much longer (31).

Another experimental study using a painful mechanical scar model did not find neuronal activation in the dorsal horn. No neuronal activation (C-fos staining), which can occur following the noxious stimuli, was found in their experiment. The activation of dorsal horn neurons depends on both mechanical stimulation force and depth of anesthesia, which could have influenced these results. They did find aberrations in the myelin sheet of the spinal nerve innervating the scarred area matching Wallerian degeneration. This can be related to the mechanical hyperalgesia that was found (46).

There exists some evidence of a more than local response after burn injuries. However, the exact location of changes in the central nervous system that cause a different sensory ability in burn patients is unclear. By testing the burn scar, the uninjured contralateral dermatome, as well as another uninjured site, a spinal segmental change can be detected. However, spinal and supra-spinal integrating systems are hard to test (16). On the other hand, there also exist arguments against a systemic effect on pain perception. Isoardo et al (11), for example, found that in patients with burns on both sides of the body, only one side was painful, which is hard to explain if the pain response in the entire body would be affected the same way.

\section{Discussion}

We performed a comprehensive systematic review on the prevalence, etiology, and pathophysiology of pain in dermal scars to give an overview of current knowledge. Although we performed a wide search in 6 databases, we cannot guarantee inclusion of all available papers with any relevance to the subject. We vastly improved our inclusion guarantee by checking titles and abstracts of all references from the included papers. Also, the liberal selection process, that enabled us to include papers with unexpected viewpoints, resulted in a large variety in available literature that made it challenging to analyze in a systematic way.

It is remarkable how little is known about the prevalence of painful scars. Most papers addressed burn scars, a specific type of scar known to give rise to pain symptoms. The only estimate of the prevalence of painful scars (excluding neuromas) in a general population with a surgical scar was $2 \%$. The prevalence of painful scars found in specific populations like burn patients or patients with pathologic scars was studied more and is much higher $(30 \%-68 \%)$. It would be valuable to study pain in scars in a design similar to that of Hoimyr et al (10) to establish whether their results in a general population are reproducible and to determine whether pain is solely a problem for burn scars, pathologic scars, and neuromas or nerve entrapment.

Although a relationship between the number of nociceptor fibers and pain sensations is plausible, various researchers question this hypothesis $(20,41,61)$. The highly variable nerve fiber densities found in scars cannot clarify this issue. However, the findings are directing towards an imbalance between non-peptidergic unmyelinated fibers (IB4-IR of N2X3-IR) and peptidergic (CGRP-IR and Sub-P-IR) fibers in painful scars. The same imbalance is also present after nerve dissection with neuropathic pain $(62,63)$. Neuropathic pain might also 
appear when epidermal fibers are directly damaged during injury. The density of the scar tissue can hamper restoring a physiologic balance between the 2 types of epidermal fibers and cause permanent symptoms $(29,33)$. Decreasing the scar adhesions, by for example lipofilling, can alleviate scar pain according to the study of Huang et al (60). Studying the nerve fiber density and balance in a clinical setting is challenging but necessary. Neuropathic pain should be objectively assessed, with extensive sensory testing and questionnaires, in order to correlate pain with nerve fiber density. Consequently, patients have to consent to supply tissue of the scar and control tissue. In addition to pan-neuronal markers, markers differentiating between peptidergic and non-peptidergic fibers should be used.

The neuroinflammatory response is most likely important in the development of painful scars. But what is the ideal level of inflammation and its perfect timing? Even if we would know this, interfering in these processes is not without risks, because factors like NGF and opioids play a role in many more processes than re-innervation and wound healing.

In general it is assumed that pain and underlying causes are local processes, as most authors use a control site within the same patient. We presented existing evidence on systemic effects of pain on a specific site on the body. Fitzgerald and Walker (58) concluded that pain early in life causes long-term effects on sensibility and pain behavior due to the developing nervous system. If ongoing neuronal development would be the sole reason for the systemic effects of pain in early life, this systemic effect would not exist in adults with complete development of the peripheral nerve system. In order to further investigate systemic effects of pain in experimental studies, separate control animals can be used. In clinical studies control tissue form another participant would, by our opinion, introduce too much heterogeneity.

\section{Conclusions}

Our conclusion is that, normotrophic scars are rarely painful (estimated at less than $2 \%$ ), while burn and pathologic scars more often lead to pain symptoms $(30 \%-68 \%)$ with high intensity (means of $1.9-6.4$ on a 10 point scale). With surgical scars, the procedure, surgical technique, and patient age are etiologic factors. For burn scars, the size and depth of the burn and post traumatic psychological disorders are important. The pain in scars, which has many characteristics of neuropathic pain, could be caused by an imbalance of peptidergic and non-peptidergic fibers in the scar area. The increased density and hard penetrability of the scar tissue may cause the different nerve fiber distribution. The latter may also be caused by ongoing neuro-inflammation that attracts and stimulates peptidergic fibers. Future research should try to confirm these theories and attempt to alter these reactions to improve the pain in the scar area, without interfering with other body systems like the immune system.

\section{References}

1. Brown BC, McKenna SP, Siddhi K, McGrouther DA, Bayat A. The hidden cost of skin scars: Quality of life after skin scarring. Journal of Plastic, Reconstructive Q A Aesthetic Surgery 2008; 61:1049-1058.

2. Niessen FB, Spauwen PH, Schalwijk J, Kon M. On the nature of hypertrophic scars and keloids: A review. Plastic and Reconstructive Surgery 1999; 104:1435-1458.

3. Nguyen DT, Orgill DP, Murphy GF. The pathophysiologic basis for wound healing and cutaneous regeneration. In: Orgill DP, Blanco C (eds). Biomaterials For Treating Skin Loss. Woodhead Publishing (UK/Europe) \& CRC Press (US), Cambridge/Boca Raton, 2009, pp 25-57.

4. Curtin C, Carrol I. Cutaneous neuroma physiology and its relationship to chronic pain. J Hand Surg (USA) 2009;
34:1334-1336.

5. Anand P. Nerve growth factor regulates nociception in human health and disease. $\mathrm{Br}$ J Anaesth 1995; 75:201-208.

6. Choi YH, Kim KM, Kim HO, Jang YC, Kwak IS. Clinical and histological correlation in post-burn hypertrophic scar for pain and itching sensation. Ann Dermatol 2013; 25:428-433.

7. Choiniere M, Melzack R, Papillon J. Pain and paresthesia in patients with healed burns: An exploratory study. J Pain Symptom Manage 1991; 6:437-444.

8. Dauber A, Osgood PF, Breslau AJ, Vernon HL, Carr DB. Chronic persistent pain after severe burns: A survey of 358 burn survivors. Pain Med 2002; 3:6-17.

9. Eishi K, Bae SJ, Ogawa F, Hamasaki Y, Shimizu K, Katayama I. Silicone gel sheets relieve pain and pruritus with clinical improvement of keloid: Possible target of mast cells. J Dermatolog Treat 2003; 14:248-252.

10. Hoimyr $\mathrm{H}$, von Sperling ML, Rokkones KA, Stubhaug A, Finnerup K, Jensen TS, Finnerup NB. Persistent pain after surgery for cutaneous melanoma. Clin J Pain 2012; 28:149-156.

11. Isoardo G, Stella M, Cocito D, Risso D, Migliaretti G, Cauda F, Palmitessa A, Faccani G, Ciaramitaro P. Neuropathic pain in post-burn hypertrophic scars: A psychophysical and neurophysiological study. Muscle Nerve 2012; 45:883-890.

12. Kehlet $\mathrm{H}$, Jensen TS, Woolf CJ. Persistent postsurgical pain: Risk factors and prevention. Lancet 2006; 367:1618-1625.

13. Lee SS, Yosipovitch G, Chan YH, Goh $\mathrm{CL}$. Pruritus, pain, and small nerve fiber function in keloids: A controlled study. J Am Acad Dermatol 2004; 51:1002-1006. 
14. Lynch DM, Harkless LB. Painful plantar scars. J Am Podiatr Med Assoc 1986; 76:681-683.

15. Maguire MF, Ravenscroft A, Beggs D, Duffy JP. A questionnaire study investigating the prevalence of the neuropathic component of chronic pain after thoracic surgery. European Journal of Cardiothoracic Surgery 2006; 29:800-805.

16. Malenfant A, Forget R, Amsel R, PapilIon J, Frigon JY, Choinière M. Tactile, thermal and pain sensibility in burned patients with and without chronic pain and paresthesia problems. Pain 1998; 77:241-251.

17. Malenfant A, Forget R, Papillon J, Amsel R, Frigon JY, Choiniere M. Prevalence and characteristics of chronic sensory problems in burn patients. Pain 1996; 67:493-500.

18. Parnell LKS, Nedelec B, Rachelska G, Lasalle $L$. Assessment of pruritus characteristics and impact on burn survivors. J Burn Care Res 2012; 33:407-418.

19. Schneider JC, Harris NL, El Shami A, Sheridan RL, Schulz JT, 3rd, Bilodeau $M$, Ryan CM. A descriptive review of neuropathic-like pain after burn injury. Journal of Burn Care \& Research 2006; 27:524-528.

20. Tey HL, Maddison B, Wang $H$, Ishiju Y, McMichael A, Marks M, Willford P, Maruzivab D, Ferdinando D, Dick J, Yosipovitch G. Cutaneous innervation and itch in keloids. Acta Derm Venereol 2012; 92:529-531.

21. Van Der Wal MBA, Offringa T, Derriks F, Van Unen E, Middelkoop E. Outcome after burns: A clinical observational study on the maturation of scar characteristics. Burns 2009; 35:S21.

22. Ward RS, Saffle JR, Schnebly WA, HayesLundly C, Reddy R. Sensory loss over grafted areas in patients with burns. Journal of Burn Care and Rehabilitation 1989; 10:536-538.

23. Widgerow AD. New advances in widespread/burn scar management. Expert Rev Dermatol 2013; 8:589-592.

24. Li-Tsang CWP, Lau JCM, Chan CCH. Prevalence of hypertrophic scar formation and its characteristics among the Chinese population. Burns 2005; 31:610-616.

25. Judkins K. Pain management in the burned patient. Pain Rev 1998; 5:133-146.

26. Yosipovitch G, Samuel LS. Neuropathic and psychogenic itch. Dermatol Ther 2008; 21:32-41.

27. Owens DM, Lumpkin EA. Diversifica- tion and specialization of toucht receptors in skin. Cold Spring Horb Perspect Med 2014; 4:a013656.

28. McArthur JC, Stockes EA, Hauer P, Cornblath DR, Griffin JW. Epidermal nerve fiber density: Normative reference range ans diagnostic efficiency. Arch Neurol 1998; 55:1513-1520.

29. Aldskogius $\mathrm{H}$, Hermanson A, Jonsson CE. Reinnervation of experimental superficial wounds in rats. Plastic and Reconstructive Surgery 1987; 79:595-599.

30. Altun V, Hakvoort TE, van Zuijlen PP, van der Kwast TH, Prens EP. Nerve outgrowth and neuropeptide expression during the remodeling of human burn wound scars. A 7-month follow-up study of 22 patients. Burns 2001; 27:717-722.

31. Anderson JR, Zorbas JS, Phillips JK, Harrison JL, Dawson LF, Bolt SE, Rea SM, Klatte JE, Paus R, Zhu B, Giles NL, Drummond PD, Wood FM, Fear MW. Systemic decreases in cutaneous innervation after burn injury. Journal of Investigative Dermatology 2010; 130:1948-1951.

32. Biyani A, Wolfe K, Simison AJM, Zakhour HD. Distribution of nerve fibers in the standard incision for carpal tunnel decompression. J Hand Surg (USA) 1996; 21:855-857.

33. Crowe R, Parkhouse N, McGrouther D, Burnstock G. Neuropeptide-containing nerves in painful hypertrophic human scar tissue. Br J Dermatol 1994; 130:444-452.

34. Hamed K, Giles N, Anderson J, Phillips JK, Dawson LF, Drummond P, Wallace $\mathrm{H}$, Wood FM, Rea SM, Fear MW. Changes in cutaneous innervation in patients with chronic pain after burns. Burns 2011; 37:631-637.

35. Henderson J, Terenghi G, McGrouther DA, Ferguson MWJ. The reinnervation pattern of wounds and scars may explain their sensory symptoms. J Plast Reconstr Aesthetic Surg 2006; 59:942-950.

36. Kwak IS, Choi YH, Jang YC, Lee YK. Immunohistochemical analysis of neuropeptides (protein gene product 9.5, substance $\mathrm{P}$ and calcitonin gene-related peptide) in hypertrophic burn scar with pain and itching. Burns 2014.

37. Nedelec B, Hou Q, Sohbi I, Choinière $M$, Beauregard G, Dykes RW. Sensory perception and neuroanatomical structures in normal and grafted skin of burn survivors. Burns 2005; 31:817-830.

38. Reynolds ML, Fitzgerald M. Long-term sensory hyperinnervation following neonatal skin wounds. Journal of Comparative Neurology 1995; 358:487-498.

39. Zhang LQ, Laato M. Innervation of normal and hypertrophic human scars and experimental wounds in the rat. Annales Chirurgiae et Gynaecologiae 2001; 90 Suppl 215:29-32.

40. Ward RS, Tuckett RP, English KB, Johansson $O$, Saffle JR. Substance $P$ axons and sensory threshold increase in burn-graft human skin. J Surg Res 2004; 118:154-160.

41. Wang C, Hah J, Mackey S, Carrol I. Quantitative sensory abnormalities in post-surgical cutaneous nerve injuries. J Pain 2010; 11:S17.

42. Bigliardi-Qi M, Gaveriaux-Ruff C, Pfaltz K, Bady P, Baumann T, Rufli T, Kieffer BL, Bigliardi PL. Deletion of $\mu$ - and $\kappa$-opioid receptors in mice changes epidermal hypertrophy, density of peripheral nerve endings, and itch behavior. Journal of Investigative Dermatology 2007; 127:1479-1488.

43. Tavee J, Zhou L. Small fiber neuropathy: A burning problem. Cleveland Clinic Journal of Medicine 2009; 76:297-305.

44. Lahteenmaki T, Waris T, Asko-Seljavaara S, Sundell B. Recovery of sensation in free flaps. Scan J Plast Reconstr Surg Hand Surg 1989; 23:217-222.

45. Scott JR, Muangman P, Gibran NS. Making sense of hypertrophic scar: A role for nerves. Wound Repair Regen 2007; 15:S27-S31.

46. Kajita Y, Suetomi K, Okada T, Ikeuchi M, Arai YCP, Sato K, Ushida T. Behavioral and neuropathological changes in animal models of chronic painful scar. J Ortop Sci 2013; 18:1005-1011.

47. Cheng B, Liu HW, Fu XB. Update on pruritic mechanisms of hypertrophic scars in postburn patients: The potential role of opioids and their receptors. J Burn Care Res 2011; 32:e118-e125.

48. Woolf CJ. Phenotypic modification of primary sensory neurons: The role of nerve growth factor in the production of persistent pain. Philosophical Transactions of the Royal Society B: Biological Sciences 1996; 351:441-448.

49. Andreev NY, Dimitrieva N, Koltzenburg M, McMahon SB. Peripheral administration of nerve growth factor in the adult rat produces a thermal hyperalgesia that requires the presence of sympathetic post-ganglionic neurones. Pain 1995; 63:109-115.

50. Nara T. Histamine and 5-hydroxytryptamine in human scar tissue. Ann Plast 
Surg 1985; 14:244-247.

51. Kaartinen IS, Valisuo PO, Bochko V, Alander JT, Kuokkanen HO. How to assess scar hypertrophy - A comparison of subjective scales and spectrocutometry: A new objective method. Wound Repair Regen 2011; 19:316-323.

52. Radtke C, Vogt PM, Devor M, Kocsis JD. Keratinocytes acting on injured afferents induce extreme neuronal hyperexcitability and chronic pain. Pain 2010; 148:94-102.

53. Taherzadeh O, Otto WR, Anand U, Nanchahal J, Anand P. Influence of human skin injury on regeneration of sensory neurons. Cell Tissue Res 2003; 312:275-280.

54. Salgado RM, Alcantara L, Mendoza-Rodriguez CA, Cerbon M, Hidalgo-Gonzalez C, Mercadillo P, Moreno LM, Alvarez-Jimenez $R$, Krotzsch E. Post-burn hypertrophic scars are characterized by high levels of IL-1(beta) mRNA and protein and TNF-(alpha) type i receptors. Burns 2012; 38:668-676.
55. Bigliardi PL, Tobin DJ, Gaveriaux-Ruff C, Bigliardi-Qi M. Opioids and the skin Where do we stand? Experimental Dermatology 2009; 18:424-430.

56. Cheng B, Liu HW, Fu XB, Sheng ZY, $\mathrm{Li} J \mathrm{~F}$. Coexistence and upregulation of three types of opioid receptors, mu, delta and kappa, in human hypertrophic scars. Br J Dermatol 2008; 158:713-720.

57. Chang PJ, Chen MY, Huang YS, Lee CH, Huang CC, Lam CF, Tsai YC. Morphine enhances tissue content of collagen and increases wound tensile strength. Journal of Anesthesia 2010; 24:240-246.

58. Fitzgerald M, Walker S. The role of activity in developing pain pathways. Proceedings of the 1oth World Congress on Pain 2003; 24:185-196.

59. Schmelzle-Lubiecki BM, Campbell KAA, Howard RH, Franck L, Fitzgerald M. Long-term consequences of early infant injury and trauma upon somatosensory processing. Eur J Pain 2007; 11:799-809.

6o. Huang SH, Wu SH, Chang KP, Cheng KI, Lee SS, Kwan AL, Yeh JL, Tsai HP,
Lin SD, Lai CS. Autologous fat grafting alleviates burn-induced neuropathic pain in rats. Plast Reconstr Surg 2014; 133:1396-1405.

61. Truini A, Biasiotta A, Di Stefano G, Leone C, La Cesa S, Galosi E, Piroso S, Pepe A, Giordano C, Cruccu G. Does the epidermal nerve fibre density measured by skin biopsy in patients with peripheral neuropathies correlate with neuropathic pain? Pain 2014; 155:828-832.

62. Duraku LS, Hossaini M, Schu "ttenhelm BN, Holstege JC, Baas M, Ruigrok TJH, Walbeehm ET. Re-innervation patterns by peptidergic Substance-P, non-peptidergic $\mathrm{P}_{2} \mathrm{X}_{3}$, and myelinated NF-200 nerve fibers in epidermis and dermis of rats with neuropathic pain. Experimental Neurology 2013; 241:13-24.

63. Kambiz S, Duraku LS, Baas M, Nijhuis TH, Cosgun SG, Hovius SER, Ruigrok TJH, Walbeehm ET. Long-term followup of peptidergic and nonpeptidergic reinnervation of the epidermis following sciatic nerve reconstruction in rats. J Neurosurg 2015; 123:254-269. 
